\title{
Urogynecology: Introducing This Subspecialty for Managing Female Pelvic Floor Dysfunction in Nepal
}

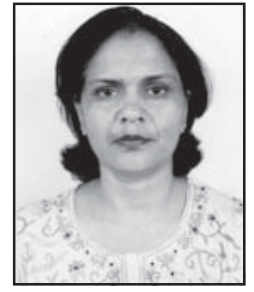

Dr. Pushpa Chaudhary Prof. NAMS
Evolution of Homosapiens from four-footed animal and acquisition of erect posture was a major breakthrough but it also resulted in the added role of weight bearing by relatively weaker pelvis. Women face consequences of pelvic floor dysfunction more as female pelvis is not only weakened by three orifices but also bear the neuromuscular injury to its ligaments, muscles as well as nerves during childbirth. In addition, deficiency of estrogen after menopause also leads to poor vascular supply and decrease in tissue strength resulting in Pelvic floor dysfunction (PFD).

Pelvic floor dysfunction (PFD) is a disorder that predominantly affects women. It is common and undermines the quality of lives of at least one third of women. ${ }^{1}$ Mechanical, neural, and endocrine disturbances all play a role in the development of PFD as it is associated with childbirth and obstetric injury, previous gynecological or anorectal surgery, aging, menopause, obesity, and conditions that produce chronic pelvic floor straining. ${ }^{1}$

Urogynecology is a subspecialty of Obstetrics and Gynecology that deals with the diagnosis and treatment of urinary incontinence and other dysfunctions of the pelvic floor. It include management of disorders such as voiding difficulties, urinary incontinence, congenital abnormalities, urinary fistulae, bladder neuropathy, genital prolapse, urgency and frequency and urinary tract infections. ${ }^{2}$ Urinary incontinence constitutes the major proportion of urogynecology and is defined by International Continence Society as ' an involuntary loss of urine which is objectively demonstrable and a social or hygienic problem.'

For assessment of genital prolapse, several prolapses grading system exists. However, the only system that has been robustly tested for both inter observer and intra observer reliability is the Pelvic Organ Prolapse Quantification system. This system defines the extent of prolapse by measuring descent of anterior, posterior and apical segments of the vaginal wall relative to the vaginal hymen. ${ }^{4}$

Urodynamic investigations are an assessment of lower urinary tract and comprised a series of tests to assess storage and voiding function of the bladder and urethra. Bladder has been described as unreliable witness as there can be discrepancy between patient's symptoms and the urodynamic test results. Three is no randomized controlled trial evidence to support mandatory urodynamic studies in patients undergoing urogynecological surgeries. ${ }^{5}$ However, RCOG Study Group recommends preoperative urodynamic, filling cystometry and pressure flow studies in all patients with suspected voiding dysfunction and if previous surgery has failed. ${ }^{6}$

Impact of pelvic floor dysfunction is profound. The social isolation caused by incontinence was found among $25 \%$ of patients delaying for more than 5 years before seeking advice. ${ }^{3}$ There are a wide range of functional problems seen with pelvic floor disorders. A dedicated team approach with fully integrated services is more effective and offers dynamic links between disciplines such as gynecologists, urologists and colorectal surgeons. A multidisciplinary approach by development of combined outpatient and joint surgical procedures and introduction of evidence-based combination therapies also facilitates appropriate and timely investigations and referrals, and better coordination of patient-centered care. ${ }^{1}$

Prevalence of pelvic organ prolapse among Nepalese

Correspondence

Prof. Dr. Pushpa Chaudhary, M.B.B.S. M.D. M.R.C.O.G. (UK)

Chief Consultant Gynecologist

Coordinator, Urogynecology unit

Paropkar Maternity and Women's Hospital,

Kathmandu, Nepal

Mobile: 9851052755

E-mail :deopuspa@gmail.com 
women is as high as $10 \%$ in general population. A clinic based study at Far western Nepal revealed that one fourth of women were attending the clinic due to problem related to pelvic organ prolapse. It is difficult to quantify other urogynecological problems such as urinary incontinence, vault prolapse etc. due to lack of data. However, more and more cases of recurrent pelvic organ prolapse and vault prolapse are expected in near future as attempts are being made to perform uterine prolapse surgery in limited theatre standards in districts hospitals of Nepal with suboptimal presurgery screening and time constraint of performing as many procedures as possible. In addition, co- existent urinary incontinence may not be addressed during prolapse surgery or it may become overt once prolapse is corrected.

Urogynecology as a subspecialty has not been developed so far in the country and skills and expertise in managing complicated prolapse with or without urinary incontinence is scarcely available. Recently, there is some attempt to establish urogynecology services in Paropkar Maternity and Women's Hospital, Kathmandu. The huge clients flow in Paropkar Maternity and Women's Hospital makes it a suitable site to develop this as service site for this subspecialty and being the centre for post graduate training a suitable institute for starting fellowship in this faculty in the long term. Modern surgical techniques for stress incontinence (Tran's obturator vaginal tape insertion) and vault prolapse (Intravaginal slingoplasty) are available now. They are relatively safe and simple, though they do carry risks of tape rejection, tape erosion and other problems. Setting an urodynamics studies equipment is challenging due to cost involved but may be made available in near future. Similarly, building up human resource and a full-fledged team is needed to provide continuity of services.
Our survey in Paropkar Maternity and Women's Hospital reflects that urinary incontinence is a hidden problem among our women but they need to know about commencement of services. Only a proportion of them will need surgical treatment but many of them will be benefited by education and conservative approach. Developing educational material and motivating media will be another activity to move the program forward... Scaling up services and training will need combined effort of the government, the hospital authority and other stakeholders and commitment of the team working in this subspecialty.

\section{References}

1. Davis K, Kumar D, Stanton SL. Pelvic Floor Dysfunction: The Need for a Multidisciplinary Team Approach. Journal of Pelvic Medicine and Surgery: Jan -Feb 2003; 9 (1): 23-36

2. Stuar L.Stanton. Classification of urogynecological disorders. Gynecology Part II Third edition edited by Robert W. Shaw, W. Patrick Soutter, Stuart L. Stanton. London. Churchil Livingstone 2003 p739

3. Norton P. MacDonald L. Sedgwick P. Stanton SL. Distress and delay associated with urinary incontinence, frequency and urgency among women. British Medical Journal 1988. 297: 1187-1189

4. Anjum Doshani, Pedrick E C Tea, Christopher J Mayne, Douglas G Tincello. Uterine Prolapse. BMJ 2007; 335:819-823

5. Gillian Fowler, David Richmond. Urodynamics: a mandatory preoperative investigation? The Obstetrician and Gynecologist 2006; 8:86-90

6. MacLean AB, Cardozo L. EDITORS. Recommendations arising from the $42^{\text {nd }}$ study group .Incontinence in women. London, RCOG press; 2002 p 433-41 